Effrosyni Lamprou, University of Poitiers, France

Freiderikos Valetopoulos, University of Poitiers, France

DOI: 10.17951/Ismll.2020.44.1.135-145

\title{
Traduire la peur : une étude contrastive
}

\author{
Translate fear: A Contrastive Analysis
}

\begin{abstract}
RÉSUMÉ
Dans cet article, nous nous intéressons à examiner la question de la verbalisation de la peur et de sa traduction du grec moderne vers le français. Les textes cibles de notre analyse sont de deux types : les traductions de traducteurs expérimentés et les traductions d'apprenants chypriotes en cours de formation. Nous étudions des données provenant de l'analyse de notre corpus de traduction et nous nous interrogeons sur la conceptualisation de l'émotion de la peur.

Mots-clés : émotion, peur, traduction, verbalisation, conceptualisation, combinatoire lexicale, équivalence
\end{abstract}

\section{ABSTRACT}

In this paper, we examine the question of the verbalization of fear and its translation from Modern Greek into French. The target texts of our analysis are of two types: translations of experienced translators and translations of Cypriot learners. We study data from the analysis of our translation corpus and we question the conceptualisation of the emotion of fear.

Keywords: emotion, fear, translation, verbalization, conceptualization, lexical combinatory, equivalence

\section{Introduction}

Comme le souligne Franzelli (2008, p. 221),

[e]n matière d'émotions, le travail d'un traducteur est particulièrement ardu, d'abord par le fait que le lexique émotionnel de différentes langues ne les rend pas aisément comparables. En outre un lexique émotionnel ou sentimental employé dans une culture donnée peut ne pas avoir d'équivalents ou être totalement ignoré dans une autre.

Partant de ce constant, nous nous intéressons à examiner la question de la verbalisation de la peur et de sa traduction. Notre point de départ sera le grec

Effrosyni Lamprou, UFR Lettres et Langues, Université de Poitiers, Campus Nord - Bâtiment A3, 1, Rue Raymond Cantel, TSA 11102. 86073 Poitiers Cedex 9, effrosyni.lamprou@univ-poitiers.fr, https:// orcid.org/0000-0001-6355-2409

Freiderikos Valetopoulos, UFR Lettres et Langues, Université de Poitiers, Campus Nord - Bâtiment A3, 1, Rue Raymond Cantel, TSA 11102. 86073 Poitiers Cedex 9, fvaletop@univ-poitiers.fr, https://orcid. org/0000-0001-8703-6230 
moderne, et nous nous intéresserons à deux types de textes : les traductions de traducteurs expérimentés du grec vers le français et les traductions d'apprenants en cours de formation, les traductions français $<>$ grec ayant déjà constitué notre objet d'analyse préalable (Valetopoulos, 2013). L'article est construit autour de trois axes principaux. Nous nous proposons, dans un premier temps, d'aborder très brièvement la question de la traduction des émotions et nous nous concentrerons par la suite sur la description contrastive de la peur. Enfin, nous étudierons des données provenant de l'analyse d'un corpus de traduction grec $<$ $>$ français et d'un corpus d'apprenants qui ont proposé la traduction d'un texte français $<>$ grec.

\section{Traduire une émotion}

Un passage de l'analyse de Panayiotou (2004, p. 13), repris par Pavlenko (2005, p. 77), nous paraît significatif du décalage qui peut exister au niveau de la conceptualisation et de l'expression d'une émotion (voir également Valetopoulos, 2016). Ainsi, un locuteur bilingue anglais-grec se livre à la confidence suivante :

Frustration is such an amazing word, the lack of it in a language is so amazing because it carries with it the word 'frustrate' to stop to block... so the outside force is carried in that word, it's not just what you feel it's the way you feel because an outside force that is blocking you and you don't have that in Greek... to a certain extent stenahorimeni if you want to use it in that extent, there you have it because the steno-horo could be from someone else although sometimes YOU make your steno-horo... so I don't know... (pp. 55-56).

Ceci nous fait penser au postulat de Vivier (2007), qui, à tort ou à raison, souligne :

L'expression orale et écrite des émotions est particulièrement difficile si l'on attend des descriptions et des identifications précises. De façon paradoxale, le langage semble peu armé pour les exprimer alors qu'existe l'intention d'en parler. Ni les traducteurs ni les interprètes n'échappent à la difficulté de la situation. Ils ne renoncent pas à franchir les barrières des langues et des cultures pour tenter de traduire des discours et des textes émotifs (p. 72).

En dehors de cette difficulté d'expression et de description, d'autres problèmes s'ajoutent quand on étudie les émotions à travers les langues. Nous pouvons citer les problèmes lexicographiques et le cas des dictionnaires bilingues, quand il s'agit de traduire des niveaux de langue différents (Lamprou, 2013), l'équivalence (Franzelli, 2008, 2011), la variabilité au niveau sémantique et syntaxique (Lamprou, 2018), les expressions idiomatiques et la métaphore (Augustyn \& Bouchoueva, 2009; Goosens, 2005; Mejri, Baccouche, Clas, \& Gross, 2003; Motsiou \& Valetopoulos, 2017), l'intensité (Lamprou, 2018; Franzelli 2013, 2011). 


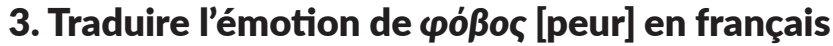

L'émotion de $\varphi o ́ \beta o \varsigma$ [peur] est définie en grec comme un sentiment désagréable et intense, provoqué par un danger ou une menace réelle ou imaginaire, alors qu'au pluriel ce mot aurait plutôt le sens d'inquiétude (voir Instituto Neoellinikon Spoudon 1998; Babiniotis, 1998). Cette définition semble focaliser plutôt sur l'intensité et la source de l'émotion, alors que les dictionnaires français définissent la peur comme un état affectif soulignant la question de la durée, de la source, de ses degrés d'intensité et de ses manifestations physiques. Nous pouvons lire dans le Trésor de la Langue Française informatisé (lemme PEUR):

État affectif plus ou moins durable, pouvant débuter par un choc émotif, fait d'appréhension (pouvant aller jusqu'à l'angoisse) et de trouble (pouvant se manifester physiquement par la pâleur, le tremblement, la paralysie, une activité désordonnée notamment), qui accompagne la prise de conscience ou la représentation d'une menace ou d'un danger réel ou imaginaire. [...]

Ainsi, si les dictionnaires grecs proposent comme synonymes de cette émotion les prédicats $\delta \varepsilon ́ o \varsigma, \sigma \varepsilon \beta \alpha \sigma \mu o ́ \varsigma$ et $\tau \rho o ́ \mu o \varsigma^{1}$, le dictionnaire TLFi renvoie à une série de prédicats qui varient sensiblement au niveau de la langue : crainte, effroi, épouvante, frayeur, terreur mais aussi frousse, pétoche, trouille, venette, les prédicats marqués " argotiques » et « populaires ». Par ailleurs, nous pouvons facilement constater qu'au niveau sémantique ces émotions n'ont pas forcément les mêmes propriétés présentant des différences surtout au niveau de l'intensité exprimée. Dans l'article de crainte, le nom peur n'apparait que dans les locutions prendre ou faire peur. Contrairement au TLFi, le Petit Robert renvoie, dans l'article de crainte, au nom peur alors que dans l'article de peur, il n'existe aucune référence au nom crainte.

Dans les différents dictionnaires bilingues grec-français (voir par exemple Lust \& Pantelodimos, 2004; Pantelodimos \& Kaïteris, 2002; Rosgovas, 2002a, 2002b; Robert \& Malamas-Robert, 2002), frayeur et appréhension. Chacune de ces traductions en français renvoie dans les dictionnaires français-grec à $\varphi o ́ \beta o \varsigma$ ainsi qu'à d'autres traductions. Ainsi, le nom appréhension est traduit par $\varphi o ́ \beta o \varsigma$ et $\delta \varepsilon ́ o \varsigma$, crainte par $\varphi o ́ \beta o \varsigma$, frayeur par $\tau \rho o ́ \mu o \varsigma$,

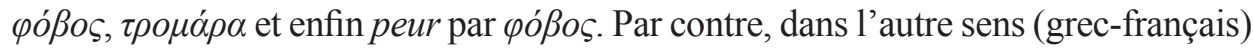

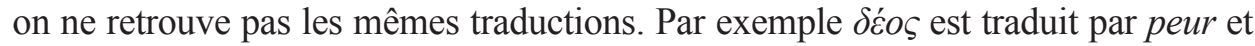

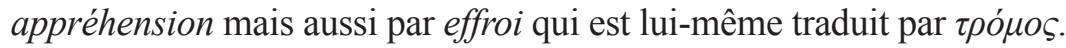

\section{Une première analyse}

Souhaitant mieux comprendre et décrire l'expression de la peur, nous avons effectué, lors d'une première étude, l'analyse des définitions lexicographiques et

1 Les traductions proposées dans le dictionnaire bilingue de Pantelodimos et Kaïteris (2002) sont respectivement effroi, respect et terreur, horreur, effroi, frayeur. 
de textes littéraires partant du français vers le grec (Valetopoulos, 2013), ce qui nous a permis de démontrer que les prédicats peur et crainte sont traduits en grec le plus souvent par le prédicat $\varphi o ́ \beta o \varsigma$. Quand le prédicat peur fait partie de la locution causative faire peur, il peut être traduit, selon le contexte, par $\varphi \circ \beta i \zeta \omega, \pi \rho o \xi \varepsilon v \omega ́$ $\varphi o ́ \beta o$ ou $\tau \rho о \mu \alpha ́ \zeta \omega$. Alors que les deux premiers dénotent plutôt l'émotion ressentie devant un danger ou une menace, le dernier se trouve sémantiquement entre deux classes, la <peur $>$ et la $<$ surprise $>$, permettant aux traducteurs d'exprimer une peur soudaine, intense et paralysante, due à une surprise négative. Nous constatons alors que les traducteurs utilisent une palette bien moins importante que les traductions qu'on peut illustrer à travers les définitions des dictionnaires :

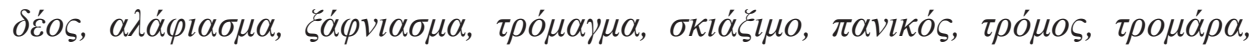

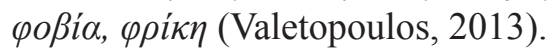

A la suite de cette première analyse, notre réflexion a été concentrée sur les prédicats morphologiquement associés (Valetopoulos, 2014). Au niveau de la combinatoire des prédicats, nous avons constaté que les adjectifs qui modifient le prédicat nominal expriment dans leur grande partie soit l'intensité soit l'origine de cette émotion. Ces deux paramètres apparaissent également au niveau des verbes supports ; ils peuvent exprimer la naissance de cette émotion, provoquée chez l'expérienceur, d'une manière progressive ou soudaine. Mais cette émotion n'est pas permanente. Contrairement au scénario de Wierzbicka (1999) qui focalise son analyse sur l'expérienceur et la prise de conscience de la peur, nous pouvons constater facilement qu'une tierce personne ou un facteur extérieur peuvent dissiper la peur, libérer le sujet de ses peurs ou, tout simplement, apaiser ses peurs (voir aussi les commentaires de Kitis, 2009).

Dans le cadre de cet article, nous nous proposons d'analyser la peur par le biais, cette fois-ci, de l'analyse des traductions du grec vers le français mais aussi à travers un corpus d'apprenants de traduction.

\section{Traduire la peur du grec vers le français}

Examinons maintenant les traductions des exemples vers le français. Dans les différentes traductions, nous pouvons relever plusieurs exemples avec craindre et avoir peur. Dans certains exemples, il est relativement facile à comprendre les raisons pour lesquelles les traducteurs ont choisi l'un ou l'autre : avoir peur dénote plutôt un état psychologique sans référence à la cause qui a provoqué ce sentiment. Le prédicat craindre en revanche est utilisé à traduire différents contextes qui ont un rapport avec la peur ou l'inquiétude, sans que le prédicat $\varphi о \beta \alpha ́ \alpha \alpha l$ 'avoir peur' soit présent dans les phrases en grec. Ainsi dans l'exemple 3, le prédicat craindre dénote l'inquiétude implicite des personnages alors que dans l'exemple 4 le même prédicat traduit l'hypothèse non réalisable, exprimée dans le contexte en grec par la structure $\lambda \varepsilon \varsigma \kappa \alpha l+$ conditionnel : 
(1)

[ZVA]

«Ils ont peur », dit-elle alors, comme si pour elle j'étais la seule personne capable de supporter un tel secret. «Ils ont peur, tu comprends ?» [...] (p. 30).

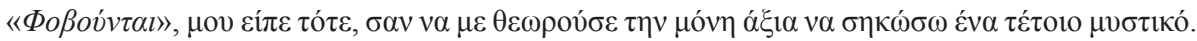

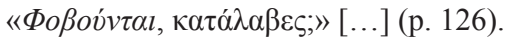

(2)

$[\mathrm{XTJ}]$

N'ayez pas peur, prenez-le dans votre main. Venez à la lumière, vous le verrez mieux (p. 332).

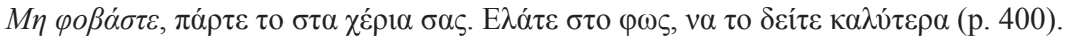

(3)

[XLM]

Revenez, il n'y a rien à craindre, absolument rien, ne soyez pas idiots ... (p. 29).

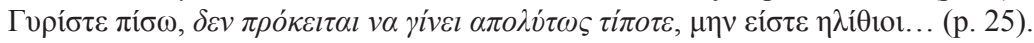

[Revenez, il ne se passera rien, ne soyez pas idiots]

(4)

[XTJ]

Il ouvrit la boîte avec mille précautions, comme s'il avait craint que le contenu ne casse la vitre et s'envole (p. 316).

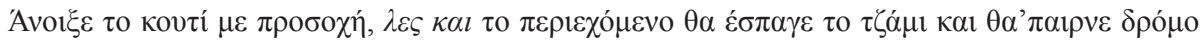
(p. 400).

[Il ouvrit la boîte avec précaution, comme si le contenu risquait de casser la vitre et de s'envoler]

Par ailleurs, le prédicat crainte permet de traduire le prédicat $\delta$ ćos du grec moderne qui renvoie à une émotion qui combine la peur et l'admiration ou le profond respect:

(5)

[XTJ]

Sous la voûte, bouche grande ouverte du Dieu de Byzance, il fut saisi d'une piété mêlée de crainte. Il eut peur de le voir descendre d'un instant à l'autre pour le mettre en pièces avec ce qui lui restait de mosaïque (p. 364).

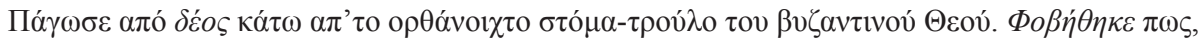

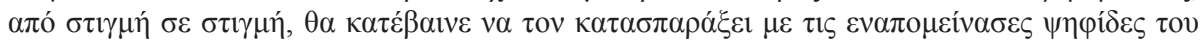
(p. 442).

En dehors de ces emplois, nous constatons que chaque traducteur peut utiliser indifféremment craindre et avoir peur dans les différentes traductions, ce qui ne nous permet pas de comprendre s'il existe une différence sémantique entre les deux verbes et dans quels contextes ils sont utilisés. Ainsi, dans la traduction La liqueur morte [XLM] on trouve plutôt la locution verbale avoir peur alors que dans l'œuvre Le vent d'Anatolie [ZVA] le traducteur utilise les deux dans les mêmes contextes syntaxiques et sémantiques : 
(6)

[ZVA]

... je me souvenais d'une jeune fille brune adossée au mur, le cou tendu vers la rue, comme si elle craignait de tourner normalement la tête ... (p. 10).

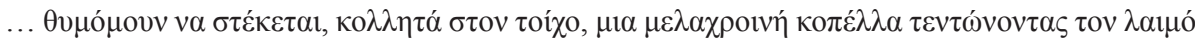

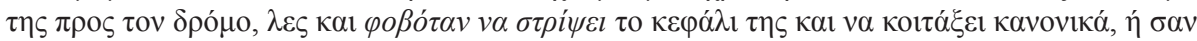

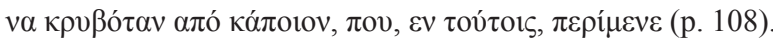

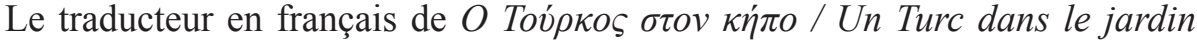
[XTJ] (exemples 7-9) semble utiliser le verbe craindre pour traduire tous les contextes dans lesquels la personne ressent ce sentiment d'inquiétude devant un danger ou devant une situation. Celui-ci ne correspond pas uniquement à la peur mais aussi à l'appréhension, à l'inquiétude :

(7)

[XTJ]

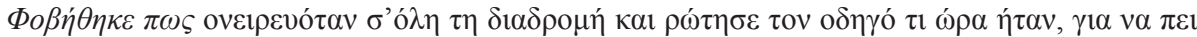
кó $\tau$ (р. 351).

Il craignit de n'avoir pas cessé de rêvasser durant les trajets et demanda au chauffeur l'heure qu'il était, histoire de dire quelque chose (p. 293).

(8)

$[\mathrm{XTJ}]$

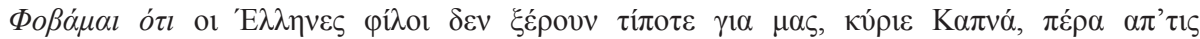

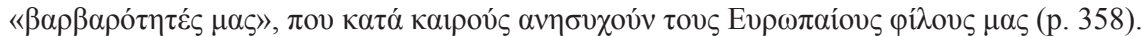

Je crains que nos amis les Grecs ne sachent rien de nous, monsieur Kapnas, en dehors de nos « barbaries » qui inquiètent de temps à autre nos amis européens (p. 299).

(9)

$[\mathrm{XTJ}]$

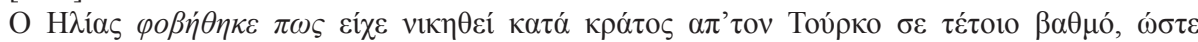

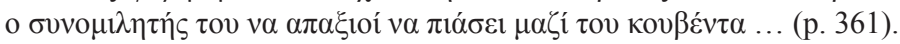

Ilias craignit d'avoir été terrassé et que le Turc ne dédaigne à présent de s'entretenir avec lui ... (p. 301).

C'est ce qu'on observe également dans tous les exemples relevés dans la traduction Le crépuscule des loups (exemples 10-11) :

[ZCL]

Une douce crainte de quelque chose qui était arrivé là-bas quand elle était beaucoup plus jeune, ou qui allait arriver d'un instant à l'autre et qui l'attendait, faisait crier les peupliers : viens, viens donc.

De vraies sapes! C'est les ciseaux que tu coupes avec des griffes pareilles! Approche, crains pas - toi qui as égorgé un agneau tout entier aujourd'hui ! 
Contrairement au prédicat craindre, la locution avoir peur est réservée aux cas où le verbe $\varphi o \beta \dot{\alpha} \mu \alpha l$ est suivi d'un complément nominal :

(12)

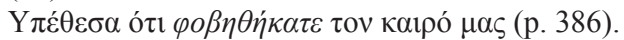

J'ai supposé que vous aviez eu peur des intempéries (p. 321).

Par ailleurs, si l'on se concentre sur les propriétés sémantiques, ce même prédicat traduit en français les différents prédicats du grec qui dénotent un état de peur ou de terreur :

[XTJ]

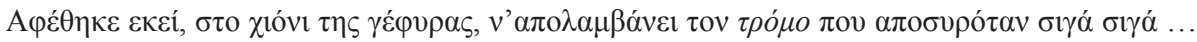
(p. 371).

Il resta sur place dans la neige du pont, jouissant de sa peur qui se retirait peu à peu, ... (p. 309).

[XTJ]

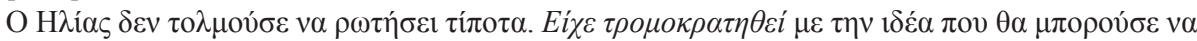

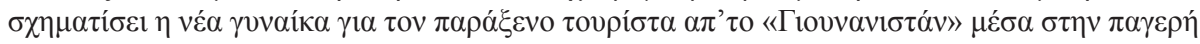

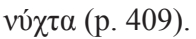

Ilias n'osait rien demander. Il avait peur de l'idée que cette jeune pouvait se faite d'un touriste de « Yunanistan » aussi bizarre que lui dans une nuit glacée (p. 339).

Il faut souligner que ces remarques correspondent à la majorité des occurrences relevées. Cependant, nous pouvons observer certains exemples qui ne semblent pas suivre nos remarques mais nous pensons que, dans ces cas-là, il s'agit d'un choix délibéré des traducteurs qui souhaitent insister plutôt sur un aspect de l'émotion de la peur. Par exemple, dans la phrase suivante, le traducteur choisit le verbe craindre suivi d'un complément humain pour insister plutôt sur l'idée du respect :

[XTJ]

X

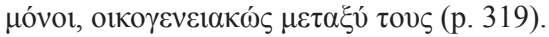

Sans Chariclée Krupp - Héraclès la craignait, la respectait et la détestait tout à la fois d'être un éteignoir; seuls et en famille (p. 269).

Enfin, il est intéressant de souligner que dans certains contextes les traducteurs semblent réinterpréter le sens du prédicat nominal $\varphi o ́ \beta o \varsigma$ et du prédicat verbal $\varphi о \beta \alpha ́ \mu \alpha l$. Ils les traduisent par des synonymes du nom peur ou de la locution avoir peur mais qui sont sémantiquement marqués tels que effrayer (par rapport à l'intensité) ou frousse (par rapport au niveau de langue) : 
(16)

[ZCL]

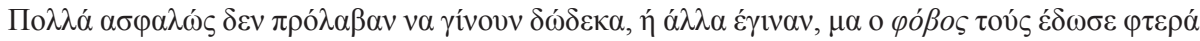
каí $\delta \rho \alpha \pi \varepsilon ́ \tau \varepsilon v \sigma \alpha \nu$ (p. 15).

Certes, bien des enfants ne purent atteindre leurs douze ans ; d'autres s'y rendirent, mais la frousse leur avait fait prendre la poudre d'escampette (p. 15).

[ZCL]

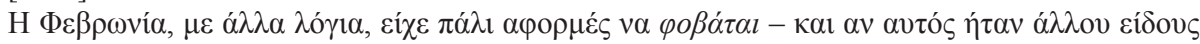

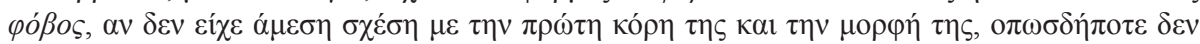

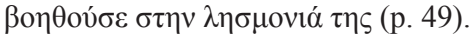

En d'autres termes, Fébronia avait de nouveaux motifs d'être effrayée - même si cette crainte était d'une autre nature, qu'elle n'avait aucun lien direct avec sa première fille et son apparence, en tout cas cela ne l'aidait pas à l'oublier (p. 49).

\section{L'expression de la peur dans un corpus d'apprenants de traduction}

Dans le cadre de notre exercice, nous avons proposé aux étudiants de quatrième année d'études de traduire certaines phrases contenant les prédicats qui nous intéressent ${ }^{2}$. A titre d'exemple, nous pouvons mentionner les extraits suivants :

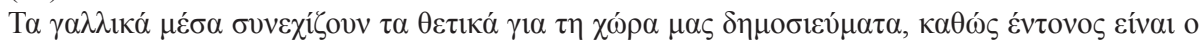

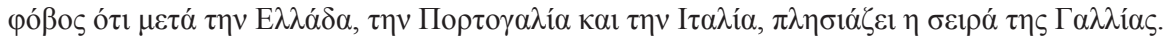

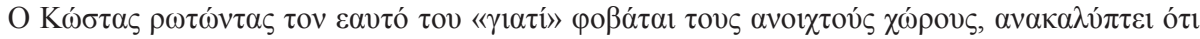

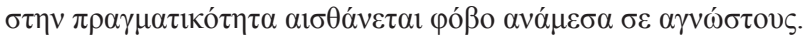

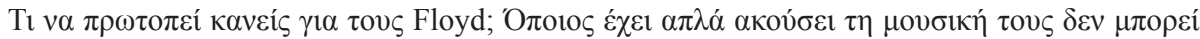

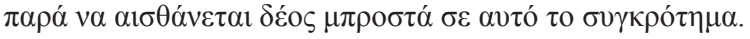

D'après les réponses proposées par les étudiants, nous pouvons constater que 12 étudiants sur 13 ont traduit systématiquement le prédicat $\varphi o ́ \beta o \varsigma$ par peur alors qu'un seul a choisi le prédicat inquiétude.

«Les medias français conduisent à garder une position positive concernant notre pays, puisqu'ils ont fortement la peur qu'après la Grèce, le Portugal et l'Italie, c'est la France qui suit » .

2 Les caractéristiques de notre groupe d'expérimentation sont les suivants : les participants ont tous le grec moderne comme langue maternelle, ils ont un niveau B1+ en français et ils ont suivi un cours de théorie sur l'activité traduisante, la procédure, les différentes théories et méthodes et un cours de pratique. 
(22)

«Les médias français continuent à transmettre des messages positifs pour la Grèce, alors que la peur est terrible à l'idée qu'après notre pays, le Portugal et l'Espagne sera le tour de la France ».

En revanche, ils sont tous unanimes sur la traduction du prédicat verbal $\varphi о \beta \alpha ́ \mu \alpha \imath$ par avoir peur :

(23)

« Kostas en tant de demander soi-même « pourquoi » il a peur des endroits ouverts, il comprend qu'il a vraiment peur d'être parmi des personnes inconnues ».

« Costas en se demandant « pourquoi » il a peur de lieux publics il se rend compte qu'en réalité il a peur quand il se trouve parmi des personnes inconnues ».

Enfin, contrairement aux deux cas précédents, nous pouvons constater un malaise des étudiants face au prédicat $\delta \varepsilon ́ o \varsigma$. Ainsi, ils ont proposé huit traductions différentes : sublime, appréciation, crainte, effroi, admiration, émerveillement et respect, ce qui illustre leur difficulté de conceptualiser la crainte en français et son rapport avec la classe sémantique de $<$ peur $>$.

«Quoi dire pour le groupe de Floyd ? Celui qui a simplement écouté leur musique, il ne peut que se sentir l'effroi en voyant ce groupe ».

"Qu'est-ce-qu'on peut dire pour Floyd ? Celui qui a entendu simplement leur musique ne peut que se sentir l'admiration face à cette bande musicale ».

\section{Discussion en guise de conclusion}

L'analyse des traductions grec $<>$ français n'a pas abouti à des conclusions permettant de construire un schéma clair comme cela a été le cas des textes français $<>$ grec. La construction syntaxique semble jouer un rôle important : le prédicat craindre traduit les contextes dans lesquels les personnages éprouvent l'émotion de la peur, également associée à l'inquiétude, à une peur intellectualisée, et la source de cette peur n'est pas exprimée. La locution avoir peur traduit les contextes où la source est exprimée. C'est la raison pour laquelle le prédicat craindre sera également associé à des prédicats tels que le respect. Contrairement aux traducteurs, nous pouvons constater que les apprenants ont une approche bien moins variée : $\varphi o ́ \beta o \varsigma$ correspond

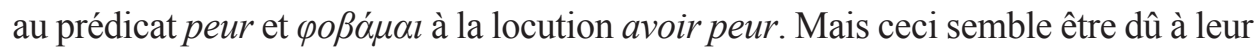
difficulté de conceptualiser cette émotion dans les deux langues.

Plusieurs remarques s'imposent. Tout d'abord, nous pensons que les dictionnaires manquent de précision ce qui conduit les apprenants à une conceptualisation hasardeuse d'une émotion relativement compliquée. Les incohérences au 
niveau des traductions dans les dictionnaires bilingues, ainsi que la description lexicographique monolingue qui ne se fonde pas sur des critères linguistiques ne permettent pas d'appréhender ces difficultés. Nous sommes convaincus que la conceptualisation de l'état émotionnel passe par l'observation et par l'utilisation de différents corpus (monolingues, bilingues-parallèles, corpus d'apprenants de traduction $)^{3}$ ce qui permettra à tous une meilleure compréhension du chapitre des émotions.

\section{References}

Augustyn, M., \& Bouchoueva E. (2009). Les collocations métaphoriques des noms de colère en français, russe et polonais. In E. Novakova, \& A. Tutin (Eds.), Le Lexique des émotions (pp. 191-205). Grenoble: Ellug.

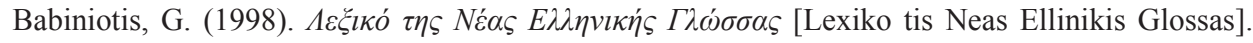
Athènes: Centre de Lexicologie.

Franzelli, V. (2008). Traduire la parole émotionnelle en sous-titrage : colère et identités. Études de linguistique appliquée, 150, 221-244.

Franzelli, V. (2011). Non mais ... tu te prends pour qui ? Le sous-titrage à l'épreuve de l'émotion. Retrieved July 15, 2019, from http://publifarum.farum.it/ezine_articles.php?id=186.

Franzelli, V. (2013). L'émotion dans tous ses états. Essai d'analyse de l'expression émotionnelle dans les films sous-titrés. In F. Baider, \& G. Cislaru (Eds.), Cartographie des émotions. Propositions linguistiques et sociolinguistiques (pp. 289-305). Paris: Presses de la Sorbonne nouvelle.

Goossens, V. (2005). Les noms de sentiment: esquisse de typologie sémantique fondée sur les collocations verbales. LIDIL : Revue de linguistique et de didactique des langues, 32, 103-122

Granger, S., \& Lefer, M.-A. (2007). Bridging the gap between learner corpus research and translation studies: The Multingual Student Translation corpus. 4th Learner Corpus Conference, Bolzano, 4-7 October 2007.

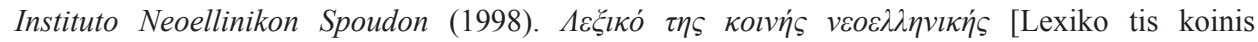
neoellinikis]. Thessaloniki: Instituto Neoellinikon Spoudon, Idrima Manoli Triantafilidi.

Kitis, E. (2009). Emotions as discursive constructs: The case of the psych-verb 'fear'. In B. Lewandowska-Tomaszczyk, \& K. Dziwirek (Eds.), Studies in Cognitive Corpus Linguistics (pp. 147-172). Frankfurt am Main: Peter Lang Verlag.

Lamprou, E. (2013). La traduction des niveaux de langue dans l'expression des sentiments : étude comparative entre le français et le grec moderne. In H. Chuquet, R. Nita, \& F. Valetopoulos (Eds.), Des sentiments au point de vue : études de linguistique contrastive (pp. 137-155). Rennes: Presses Universitaires Rennes.

Lamprou, E. (2018). Définir la « colère » en français et en grec : étude basée sur un corpus de textes sous-titrés. In R. Nita, \& F. Valetopoulos (Eds.), L'expression des sentiments : De l'analyse linguistique aux applications (pp. 83-94). Rennes: Presses universitaires Rennes.

Lust, C., \& Pantelodimos D. (2004). Dictionnaire français-grec moderne. Athènes: Librairie Kauffmann.

Mejri, S., Baccouche, T., Clas, A., \& Gross G. (Eds.) (2003). Traduire la langue Traduire la culture. Paris: Sud éditions.

Motsiou, E. , \& Valetopoulos, F. (2017). La phraséologie de la peur et de la surprise en russe et en grec [H

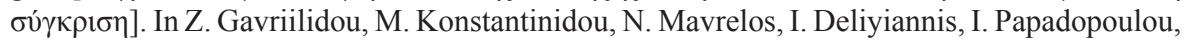

\footnotetext{
3 Voir Multilingual Student Translation corpus (MUST), Granger \& Lefer (2007).
} 
\& G. Tsomis (Eds.), Identities: Language and Literature: Proceedings of the International Conference on the Occasion of the $20^{\text {th }}$ Anniversary of the Department of Greek of D.U.TH. (pp. 254-275). Kavala: Saïta. Retrieved July 1, 2019, from http://www.saitapublications. gr/2017/08/ebook.175.html?m=1.

Panayiotou, A. (2004). Bilingual emotions : The untranslatable self. Estudios de Sociolingüistica, $8(1), 1-19$.

Pantelodimos, D., \& Kaïteris C. (2002). Dictionnaire grec-français. Athènes: Librairie Kauffmann. Pavlenko, A. (2005). Emotions and Multilingualism, Cambridge: Cambridge University Press.

Robert, J.-P., \& Malamas-Robert M. (2002). Dictionnaire français-grec moderne. Athènes: Efstathiadis.

Robert, P. (2000). Le nouveau petit Robert de la langue française. Paris: Petit Robert.

Rosgovas, Th. (2002a). Dictionnaire grec-français. Leonidio: Editions Rosgovas.

Rosgovas, Th. (2002b). Dictionnaire français-grec. Leonidio: Editions Rosgovas.

Valetopoulos, F. (2013). Définir la peur et la surprise en grec moderne et en français. In H. Chuquet, R. Nita, \& F. Valetopoulos (Eds.), Des sentiments au point de vue : études de linguistique contrastive (pp. 95-116). Rennes: Presses universitaires Rennes.

Valetopoulos, F. (2014). Décrire l'état psychologique de < peur>. In Z. Gavriilidou, \& A. Revithiadou (Eds.), Mélanges offerts à Anna Anastassiadis (pp. 165-178). Kavala: Saïta.

Valetopoulos, F. (2016). Quand les apprenants parlent de leurs émotions : une étude des adjectifs subjectifs à travers les corpus d'apprenants. In A. Krzyżanowska, \& K. Wołowska (Eds.), Les émotions et les valeurs dans la communication I (pp. 55-66). Frankfurt am Main, Bern, Bruxelles, New York, Oxford, Warszawa, Wien: Peter Lang Verlag.

Vivier, J. (2007). La traduction des textes émotifs : un défi paradoxal. Meta, 52(1), 71-84.

Wierzbicka, A. (1999). Emotions across Languages and Cultures: Diversity and Universals. Cambridge: Cambridge University Press.

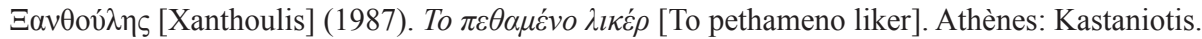

[XLM] Xanthoulis, Y. (1991). La liqueur morte (L. Rotis, Trans.). Paris: Hatier.

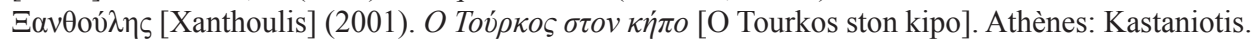

[XTJ] Xanthoulis, Y. (2005). Un Turc dans le jardin (F. Lozet, Trans.). Paris: Actes Sud.

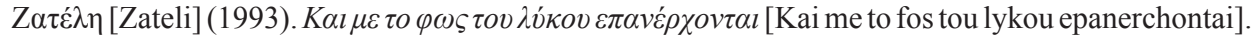
Athènes: Kastaniotis.

[ZCL] Zatèli, Z. (2001). Le crépuscule des loups (J. Bouchard, Trans.). Paris: Roman Seuil.

[ZVA] Zatèli, Z. (2012). Le vent d'Anatolie (M. Volkovitch, Trans.). Paris: Quidam Editeur. 